\title{
Searching for Exotic Signals with the NOvA Experiment
}

\author{
Peter Filip, for the NOvA Collaboration ${ }^{a, *}$ \\ ${ }^{a}$ Institute of Physics, Czech Academy of Sciences, \\ Na Slovance 1999/1, Prague 182 00, Czech Republic \\ E-mail: filip@fzu.cz
}

\begin{abstract}
Two highly segmented NOvA scintillation detectors, separated by $810 \mathrm{~km}$ and located in the path of the NuMI neutrino beam produced at Fermilab, are optimized to study the neutrino oscillation phenomenon. At the same time, the larger Far Detector with 4,000 $\mathrm{m}^{2}$ surface area is capable to search for the low-mass magnetic monopoles, and to monitor the flux of the high-multiplicity cosmic ray showers. The smaller Near Detector, located $100 \mathrm{~m}$ underground at Fermilab, is able to search for the presence of possible elusive Dark Matter particles in the high-intensity NuMI neutrino beam, and to study the seasonal variations of multi-muon cosmic flux component. Both NOvA detectors serve in combination as a powerful supernova neutrino detector, and allow for the multi-messenger signal searches in coincidence with LIGO/Virgo gravitational wave events. A survey of the most recent NOvA results on these topics is presented.
\end{abstract}

\footnotetext{
*** The European Physical Society Conference on High Energy Physics (EPS-HEP2021), *** *** 26-30 July $2021 * * *$

*** Online conference, jointly organized by Universität Hamburg and the research center DESY ***
}

\footnotetext{
${ }^{*}$ Speaker
} 


\section{NOvA Detectors and Triggers}

The NOvA experiment is optimized to measure the oscillation parameters of the muon (anti)neutrinos (using the NuMI beam at Fermilab) into electron (anti)neutrinos at $810 \mathrm{~km}$ distance from the neutrino beam origin. Two large segmented plastic detectors of the size $4 \times 4 \times 16$ meters and 16x16x60 meters, comprising 20193 and 344064 scintillator cells, were built to observe the charged current (CC) neutrino interactions in the liquid oil-based scintillator medium [1].

A high-intensity off-axis $(\sim 2 \mathrm{GeV})$ component of the NuMI neutrino beam strikes the Near (ND) and Far (FD) NOvA detectors for a $10 \mu \mathrm{s}$ (spill) time interval every 1.33 seconds. Outside of the NuMI spill trigger, the live photo-sensor data (stored in the computer memory of the NOvA DAQ cluster) are continuously scanned for the charged (cosmic) tracks, and sophisticated algorithms search for the exotic magnetic monopoles, multi-muon showers, low-energy (anti)neutrino bursts from the supernovas, or for the signals possibly associated with Gravitational waves, using a flexible set of data-driven triggers.

\section{Upper Limit on the Flux of Magnetic Monopoles}

Magnetic monopoles, arriving at NOvA detectors as an exotic component of cosmic rays, could be easily distinguishable in the Far Detector data, from the relativistic $\beta=v / c \approx 1$ muons or from the slow muons (decelarated and stopped in detector medium), as long, straight, moderately slow $\left(\beta>3 \times 10^{-4}\right)$ tracks, with a large $(d E / d x)$ ionisation. Dedicated slow-monopole data-driven trigger has been designed to search for the slow monopole tracks in the FD data. Utilizing a typical single-hit timing resolution of $\sim 20 \mathrm{~ns}$, an upper limit on the flux of magnetic monopoles was obtained [2] as shown in Fig.1 (left panel).

The surface location of the NOvA FD means lower-mass $M>5 \times 10^{8} \mathrm{GeV}$ monopoles are detectable in contrast to $M>10^{10} \mathrm{GeV}$ limited MACRO and ANTARES data. The extension of NOvA analysis to fast $\left(10^{-3}<\beta<1\right)$ monopoles, with full 12 years data sample, will allow us to extend the upper limit as shown in Fig. 1 (right panel).
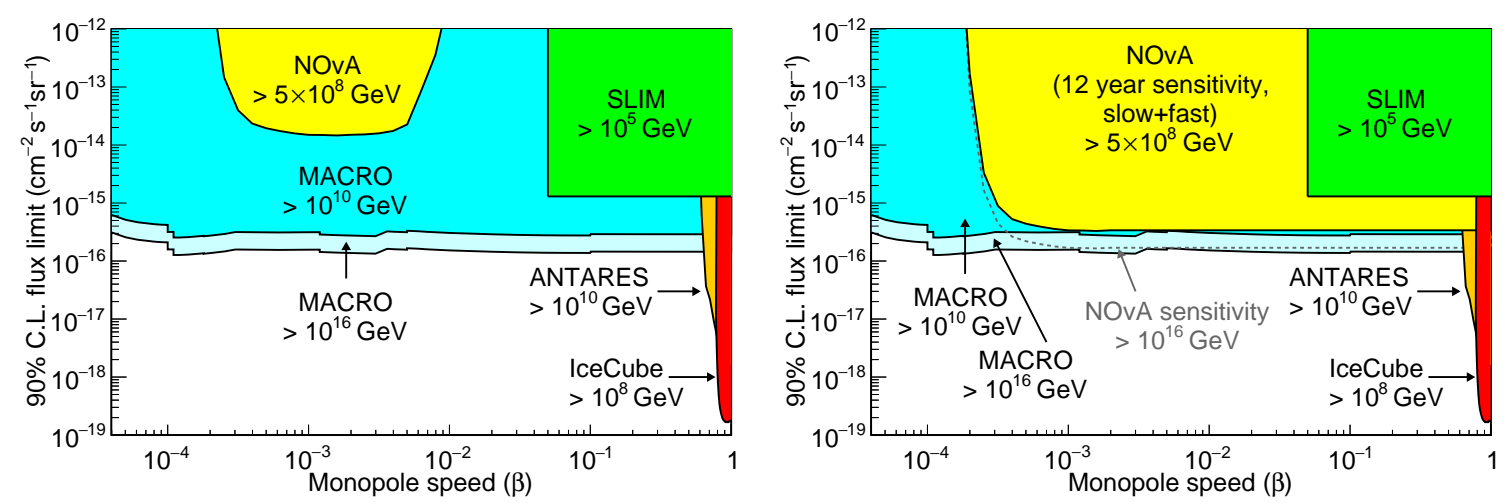

Figure 1: Upper limit on the flux of $\left(\beta \approx 10^{-3}\right)$ slow magnetic monopoles obtained from the NOvA FD data [2] in comparison with other experiments. Sensitivity of the current slow monopole analysis for the complete 12 year (2015-2026) data, and supplemented with the Fast Monopole trigger, is shown in the right panel. 


\section{Search for Sterile Neutrino Mixing and Light Dark Matter}

Far Detector data accumulated during the NuMI spill (time window) are scanned for the Neutral Current (NC) neutrino interactions, which do not depend on the neutrino $(e, \mu, \tau)$ flavour. The total flux of active neutrinos in the NuMI beam at the Far Detector location can thus be analyzed for a statistically significant deficit, possibly caused by the active $\leadsto$ passive (sterile) neutrino transitions in $810 \mathrm{~km}$ long beam path. So far, a significant deficit (due to sterile neutrino mixing) has been observed neither in the neutrino beam [3] nor in the antineutrino beam data [4].

Data from the Near Detector accumulated during the NuMI spill time window can be used to search for the possible Lightweight Dark Matter (LDM) presence in the NuMI beam, which could manifest itself as an excess of the elastic scattering events in a specific $E_{e} \theta^{2}$ kinematical region (here $\theta$ is the electron shower angle and $E_{e}$ shower energy). No such specific excess has been observed in the elastic neutrino-electron scattering NOvA-ND data [5] and a tentative upper limit on LDM production in the NuMI target has been inferred [6] within the theoretical analysis.
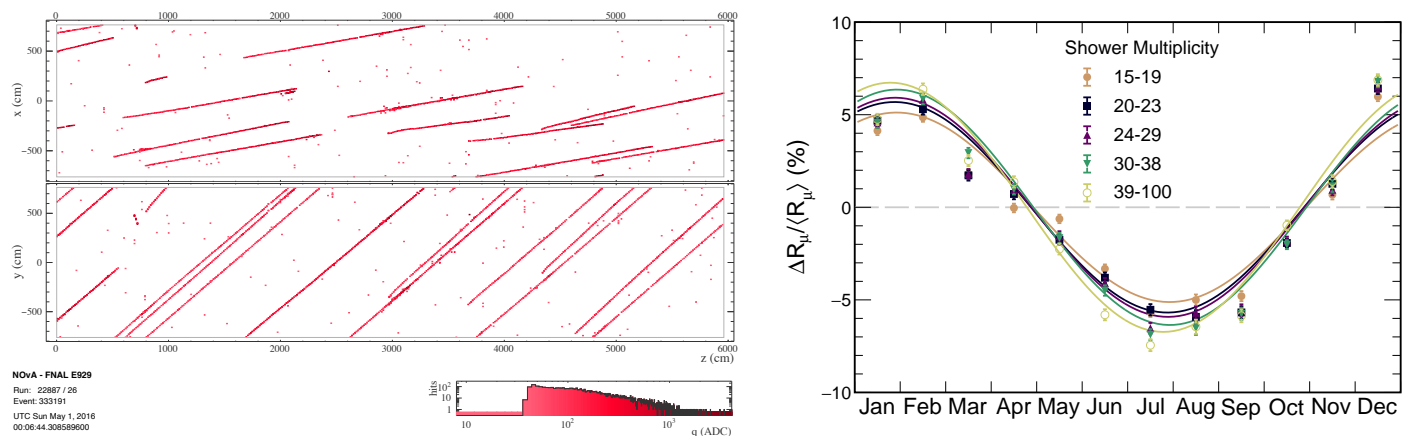

Figure 2: Event display of a multi-muon shower (left panel) in the NOvA-FD, and seasonal variation of the multi-muon shower flux [9] for different muon multiplicities observed in the Far Detector (right panel).

\section{Multi-Muon Showers}

Seasonal variation of cosmic multi-muon showers is the subject of scientific interest, also because the magnitude of the observed winter/summer multi-muon flux variation (see Fig. 2) is $2 \mathrm{x}$ larger than the most recent MC simulations predict [7]. Analysis of the NOvA cosmic data [8] from the Near (underground) Detector confirm the phase (winter maximum) and the magnitude of the seasonal multi-muon flux oscillation measured previously by the MINOS-ND.

A dedicated data-driven trigger is used to record the Far Detector cosmic data whenever the total ADC sum in the NOvA live data stream exceeds the threshold indicating a large multi-muon cosmic shower has reached the Far Detector (which is exposed to intense $\sim 130 \mathrm{kHz}$ flux of cosmic muons). New results from such triggered Far Detector cosmic showers data [9] also show the large magnitude and winter maximum of the multi-muon flux (see Fig. 2), and additionally, suggest a zenith-angle dependence of the multi-muon flux seasonal oscillation is present in data. Such observations may allow one to clarify the physics mechanisms primarily responsible for the varying probability of the large multi-muon showers generation in the atmosphere. 


\section{GW-associated Multi-messenger Signals}

The pioneering discovery of Gravitational Waves (GW) by the LIGO/Virgo Collaboration (LVC) has allowed one to search for the hints of neutrino bursts or cosmic ray flux variations originating from the processes taking place in the vicinity of two heavy objects merging into one massive body. The NOvA DAQ setup (ND and FD) is connected to LVC public alerts and allows for 45 seconds of continuous data to be saved for further data analysis [10], if timely alert is received up to 20 minutes after GW event. Moreover, $550 \mu s$ data fragments from the Far Detector (from 10 $\mathrm{Hz}$ pulser trigger) are analyzed within 1000 seconds time interval centered around the GW events.

No hints of the neutrino burst or any statistically significant excess of cosmic ray flux has been observed in $32+5$ (FD) and $40+7$ (ND) continuous readouts [10] from NOvA detectors. The Far Detector data allowed us to put median upper limit of $F<7 \times 10^{10} \mathrm{~cm}^{-2}$ at $90 \%$ C.L. [10] on the flux of supernova-like neutrinos, possibly associated with 40 selected GW-events observed by LVC.

\section{SN Neutrinos and other Exotic Signals}

The NOvA DAQ system is connected to KamLAND pre-supernova and SNEWS alert systems, and additionally, the internal software trigger [11] allows us to save continuous FD + ND data if a hint of the arriving (anti)neutrino burst from the core-collapse supernova is spotted in the NOvA live data stream. This internal trigger was setup to have one false alert per 7 days (on average), and allows NOvA to be sensitive to a supernova occurring within $10 \mathrm{kpc}$ from Earth, resulting in $23 \%$ $(49 \%)$ probability to detect a supernova event from a $9.6 \mathrm{M}_{\odot}\left(27 \mathrm{M}_{\odot}\right)$ progenitor star in our galaxy.

Description of other interesting ongoing analyses of NOvA data (e.g. of the the upward-going muons directionally correlated with the Sun position and search for the cosmic ray flux excursions associated with solar flares and GLE events) had to be skipped here due to the space restrictions.

\section{References}

[1] D. Ayres, et al., The NOvA Technical Design Report 2007-01, DOI:10.2172/935497.

[2] M.A. Acero et al., Physical Review D103, 012007 (2021).

[3] M.A. Acero et al., Physical Review D 96, 072006 (2017).

[4] M.A. Acero et al., arXiv 2106.04673 (2021) accepted by Phys. Rev. Lett.

[5] J. Bian, for the NOvA Collaboration, arXiv:1710.03428 (2017).

[6] P. deNiverville and C. Frugiuele, Physical Review D99, 051701R (2019).

[7] T.K. Gaisser and S. Verpoest, Astroparticle Physics 133, 102630 (2021).

[8] M.A. Acero et al., Physical Review D99, 122004 (2019).

[9] M.A. Acero et al., Physical Review D104, 012014 (2021).

[10] M.A. Acero et al., Physical Review D101, 112006 (2020) and D104, 063024 (2021).

[11] M.A. Acero et al., Journal of Cosmology and Astroparticle Physics 10, 014 (2020). 